\title{
Explaining outcome type interactions with frame: Aspiration level and the value function
}

\author{
AMber N. BLOOMFIELd \\ DePaul University, Chicago, Illinois
}

\begin{abstract}
Research on framing effects has revealed cases where the type of outcome at risk (e.g., human lives vs. animal lives) affects the magnitude of the framing effect. Some authors have appealed to the shape of the value function as predicting when framing effects will occur: The more valuable the outcome type, the more nonlinear its value function, and the larger the resulting framing effect (Levin \& Chapman, 1990). However, having a more or less nonlinear value function cannot explain situations in which participants strongly prefer the same option in both frames. Another factor that may be at work in these types of outcome effects is an aspiration level (AL; Lopes, 1987; Schneider, 1992), which determines how acceptable the options are and combines (or competes) with the risk attitude encouraged by frame. The results described here indicate that differences in the shape of the value function between outcome types are evident but are inconsistent between framed losses and gains, though nonlinearity in the value function can be increased with a manipulation that also encourages framing effects. The results also demonstrate that an $\mathrm{AL}$ can lead to the same predominant risk preference in the positive and negative frame. These findings indicate that the shape of the value function and the AL each play a role in outcome type interactions with frame, and in some cases, a combination of the two factors may be at work.
\end{abstract}

In framing effects, objectively identical outcomes described as gains (positive frame) or losses (negative frame) typically produce risk attitudes that are risk averse for framed gains and risk seeking for framed losses. For instance, the Asian disease problem (Tversky \& Kahneman, 1981) described a situation where a disease is expected to infect 600 people. The two options for dealing with the disease were as follows.

\section{Positive frame}

If Program A is adopted, 200 people will be saved.

If Program B is adopted, there is a $1 / 3$ probability that 600 people will be saved and a $2 / 3$ probability that no people will be saved.

\section{Negative frame}

If Program C is adopted, 400 people will die.

If Program $D$ is adopted, there is a $1 / 3$ probability that nobody will die and a $2 / 3$ probability that 600 people will die.

In the original study, $72 \%$ of participants chose Program A, the certain option, in the positive frame, but $78 \%$ of participants chose Program D, the risky option, when the options were in the negative frame. Tversky and Kahneman explained the framing effect through two factors: the shape of the value function (concave downward for gains and concave upward for losses) and the reference point established by the frame, which leads options to be seen as gains in the positive frame and as losses in the negative frame. The shape of the value function is believed to establish the risk attitude for the outcome domain (risk averse for gains and risk seeking for losses; Kahneman \& Tversky, 1979).

Although framing effects are robust, there are also numerous studies in which these effects have not occurred. In Levin and Chapman (1990), participants failed to show framing effects for AIDS patients when the groups at risk were "devalued" (e.g., intravenous drug users) and preferences in both frames were split between the two options: $50 \%$ choice of the risky option in the positive frame, $39 \%$ in the negative frame. Wang, Simons, and Bredart (2001) found similar results with a scenario featuring 6 billion extraterrestrial lives: $52 \%$ choice of the risky option in the positive frame, $52 \%$ in the negative frame. Wang et al. maintained that their participants simply did not care enough about extraterrestrial lives to be vulnerable to framing effects. Levin and Chapman argued that their failure to find a framing effect for "devalued" groups arose from participants' having a more linear value function for less valuable outcomes (see also Fagley \& Miller, 1997). Outcome types for which people have near-linear value functions are less likely to produce framing effects because each life saved or lost increases or decreases subjective value by the same amount, ${ }^{1}$ such that the gain frame fails to inspire risk aversion and the loss frame does not excite risk seeking.

Bloomfield (2006) examined the effects of frame for scenarios describing human or animal victims. Although the human scenario produced framing effects, no signifi-

A. N. Bloomfield, abloomfi@depaul.edu 
cant framing effects were shown for animal life scenarios, and participants preferred the certain option in both frames. In another condition, Bloomfield (2006) introduced a picture manipulation (a display of unique pictures and names, one corresponding to each victim) to increase caring for the victims. For animal scenarios, framing effects appeared in the pictures and names condition; for the human scenario, however, this manipulation led to overall preference for the risky option in both frames. Although the effect of pictures and names for the animal scenarios suggests that this measure may have increased nonlinearity in a previously linear value function, the effect of this manipulation for the human life scenario is more difficult to interpret. For this manipulation to eliminate the framing effect through altering the shape of the value function, the value function for human life would have to have been more linear in the pictures and names condition.

Aspiration level (AL), part of Lopes's (1987) securitypotential/aspiration theory, offers another explanation for why framing effects occur for some types of outcomes but not others. People may have an AL that influences how acceptable an option is (Lopes, 1987). For instance, if a student needs $\$ 400$ to make her rent, a gamble offering this amount would be more attractive than one offering less, even if the odds of the first were unfavorable. Schneider (1992) argued that the AL in framing scenarios is anchored and adjusted from the reference point. Because the reference point differs between frames (e.g., "0 lives lost" for losses and "0 lives saved" for gains), the adjustment for the positive frame tends to lead to a lower AL that is met by the certain option, whereas the adjustment for the negative frame leads to higher AL that is not met by the certain option (Schneider, 1992). ${ }^{2}$ For example, an AL of 200 would lead to an adjustment in the positive frame to "200 saved," whereas the same AL in the negative frame would lead to "no more than 200 lost." Schneider argued that it is the AL, not the shape of the value function (and the resulting risk attitude) per se, that leads to choice of the certain option in the positive frame, and of the risky option in the negative frame. ${ }^{3}$

Schneider's (1992) description of how an AL is set through anchoring and adjustment is one way in which AL could influence choice in framed scenarios. However, an AL may also arise from beliefs about the group at risk. When a specific outcome level is important, such as not wanting to lose any members of a human group (Wang, 1996), or wanting to maintain a number sufficient for breeding for an animal group, the AL would be of the same magnitude in both frames ${ }^{4}$ and would encourage choice of the same option. This account of AL in framed scenarios finds some support in the literature. Schneider found that participants showed a majority preference for the risk averse option in both frames when the scenario described the last 1,200 members of a species (roughly $81 \%$ choice of the certain option). In contrast, Wang and Johnston (1995) found a majority preference for the risky option in both frames for small human groups. If the participants' AL for saving/not losing animal life in Schneider's study was met by the certain option, and the one for humans in Wang and Johnston's study was not met by the certain option, this would explain why participants have the same preference in both frames and failed to show an effect of frame.

Differences in ALs and nonlinearity of the value function between outcome types each offer partial explanations of the interactions between outcome and frame discussed above. Differences in nonlinearity of the value function could account for why framing effects appear for some outcome types but not for others; however, there is no direct evidence that experimental manipulations, such as varying the outcome type (e.g., human vs. animal life) or presenting pictures and names of the victims prior to choice alter the value functions in the manner posited by this explanation. Experiment 1 examined potential differences in the shape of the value function across outcome types and across picture conditions. An AL, on the other hand, can explain a preference for the same option in both frames and no framing effect, but there is no evidence showing that people will disregard the risk attitude encouraged by framing in favor of serving an AL. Experiment 2 assessed the effects of the introduction of an $\mathrm{AL}$ on choices for framed gains and losses.

\section{EXPERIMENT 1}

Experiment 1 examined value functions ${ }^{5}$ for humans and animals to address two primary questions. Does the value function for human life and that for a less valued outcome type like grizzly bears differ in nonlinearity? To account for Bloomfield's (2006) results, the value function for human life should be more nonlinear than the value function for bears. Also, does a manipulation that encouraged framing effects in past studies change the shape of the value function? In order to support a purely value-function-based account for the effects of pictures and names on animal scenarios in Bloomfield (2006), pictures of animals should make the value function more nonlinear. Note also that, in order to support a value-function-based account for the effects of pictures and names for human life, the pictures of humans must lead to a more linear value function.

\section{Method}

Participants. One-hundred four undergraduates received partial course credit for participating in this experiment. One participant's data were excluded because of computer malfunction, and the data of 15 additional participants were excluded because they attempted to exit the experiment quickly by alternating back and forth between options or choosing the same option in each trial.

Materials and Procedure. Participants responded to a series of pairwise choices for grizzly bears and human lives involving 11 certain outcomes for each type of life. Type of life was a within-subjects variable. Participants received only positively or negatively framed options. In both frames, participants were told that 600 bears/people were at risk, and to treat each pair of options as though they were the only options available. Indifference points for each certain outcome were calculated from participants' responses in the manner described below. The order of presentation for the bear and human stimuli sets was randomized; also, within each type of life, whether the certain values started at the highest (200 for gains and 400 for losses) and descended or at the lowest (4 for both losses and gains) and ascended was randomly determined by the computer. Half the participants in each framing condition saw displays of pictures with names (pics condition) and the other half saw colored squares (neutral condition) 
periodically while making choices. The pictures were appropriate to the type of life in the current choice set and were taken from those used in Bloomfield (2006). Examples are shown in Figure 1.

Assignment to conditions was randomized. Of the 104 participants included in the analyses, 27 received the negative-frame/ pics condition, 26 received the negative-frame/neutral condition, 26 received the positive-frame/pics condition, and 25 received the positive-frame/neutral condition.

Indifference points between risky and certain options were obtained by adjusting the nonzero value associated with the risky option up or down in response to the participants' selections. For losses, zero dying was always associated with the $1 / 3$ probability in the risky option, and zero being saved was always associated with the $2 / 3$ probability for gains. At first, the outcome value of the two options was equal (e.g., 100 people saved vs. $1 / 3$ chance of saving 100 people, $2 / 3$ chance of saving no one). For participants in the positive frame, choices of the certain option resulted in increases in the nonzero value of the risky option (i.e., the outcome of the risky option started getting better); choices of the risky option resulted in decreases in the nonzero value of the risky option. For the participants in the negative frame, a choice of the risky option resulted in an increase in the value of the risky option (i.e., the cost of failure if the risky option fails became worse); choices of the certain option resulted in decreases in the risky option's value.

Pairs of options following selections of the certain option in the positive frame continued until the risky option's value reached 600 (the maximum value, because 600 lives were at stake in all cases); those involving choices of the risky option continued until the nonzero value of the risky option equaled what it had been the last time the certain option was chosen over it. For losses, choices of the risky option resulted in increases in the risky option's value until 600 was reached; choices of the certain option led to decreases in the risky option's nonzero value until its value was the same as it had been the last time the risky option was chosen over the certain option. Table 1 shows a hypothetical choice series for a certain gain of 100 . Bold font indicates the chosen option. In this case, the participant's subjective value for the certain outcome of 100 lives saved was approximately equal to the participant's subjective value for a gamble with a $1 / 3$ chance of saving 242.5 people, and a $2 / 3$ chance of saving no one. In other words, the indifference point for the value of 100 was $(245+240) / 2 *(1 / 3)+0 *(2 / 3)=80.8$, indicating a risk-seeking preference (i.e., the risky option's expected value, the value of each outcome offered by the risky option multiplied by its probability, was lower than the value of the certain option).

\section{Results}

Analyses included nine indifference points for each participant. ${ }^{6}$ Figures $2 \mathrm{~A}$ and $2 \mathrm{~B}$ show the responses for participants in the pics and neutral conditions for grizzly bears for the negative frame and positive frame conditions, respectively. Figures 3A and 3B show the results for human life. On each figure, risk neutrality is represented by a solid line: Responses above this line are risk averse and those below it are risk seeking. Table 2 also displays the average indifference points and standard errors for each condition, frame, and type of life.

The first question is whether there is a difference in nonlinearity for the value functions for the two types of life in the neutral condition. Comparing the black diamonds on Figures $2 \mathrm{~A}$ and $3 \mathrm{~A}$ with those on Figures $2 \mathrm{~B}$ and $3 \mathrm{~B}$ reveals no differences in nonlinearity: The functions are concave downward across both types of life for framed gains and concave upward for framed losses, consistent with the value function described in prospect theory (Kahneman \& Tversky, 1979). To statistically investigate differences in nonlinearity between the functions, Stevens's (1961) law was fit to each. The equation is $(X)=a *(X-\text { reference point })^{b}$ for gains, $(X)=-(a) *(\text { reference point }-X)^{b}$ for losses, with separate $a$ and $b$ parameters calculated for losses and gains. The $a$ parameter captures the sensitivity to changes in framed gains or losses (i.e., the slope of the function), and the $b$ parameter scales the concavity of the function: The smaller the $b$ parameter, the more nonlinear the function. Table 3 displays the parameters for framed losses and gains for both outcome types, and for the pics and neutral conditions.

The $b$ parameters for framed losses for the human and bear functions differed significantly from $1[t(25)=$ $26.00, p<.001$, for bears, and $t(25)=37.94, p<.001$, for humans]. The difference between the $b$ parameters for the negative frame functions was significant $[t(25)=3.70$, $p<.01]$. When no pictures were presented, the function for framed losses was more nonlinear for humans than for bears. Similar analyses were done for the positive frame neutral functions: $b$ parameters for both functions were significantly different from $1[t(24)=23.80, p<.001$, for bears, and $t(24)=10.34, p<.001$, for humans] and the difference between the $b$ parameters for the two functions was also significant $[t(24)=5.42, p<.001]$. The framed gains function for bears was actually more nonlinear than that for humans when no pictures were presented. These findings show that, even with no pictures presented, the functions for framed losses and gains were significantly nonlinear for both types of life, and despite differences between outcome types, human life functions did not have consistently greater nonlinearity.

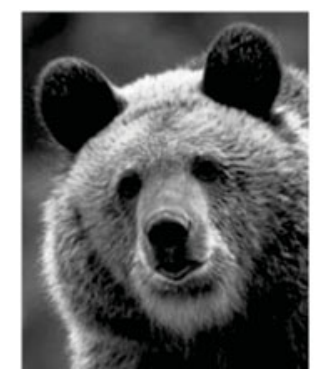

Terrance

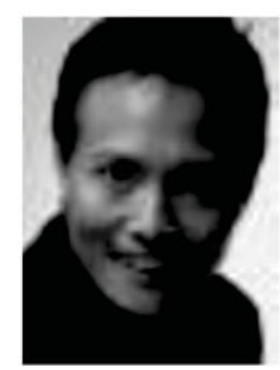

Alex

Figure 1. Examples of pictures and names. 
Table 1

Example of a Possible Choice Sequence for Gains

100 people will be saved. OR There is a $1 / 3$ chance that 100 people will be saved and a $2 / 3$ chance that none of the people will be saved. 100 people will be saved. OR There is a $1 / 3$ chance that 150 people will be saved and a $2 / 3$ chance that none of the people will be saved. 100 people will be saved. OR There is a $1 / 3$ chance that 200 people will be saved and a $2 / 3$ chance that none of the people will be saved. 100 people will be saved. OR There is a $1 / 3$ chance that 250 people will be saved and a $2 / 3$ chance that none of the people will be saved. 100 people will be saved. OR There is a 1/3 chance that 245 people will be saved and a $2 / 3$ chance that none of the people will be saved. 100 people will be saved. $\quad$ OR There is a $1 / 3$ chance that 240 people will be saved and a $2 / 3$ chance that none of the people will be saved.

Note-Bolded options are the chosen options.

The second question addressed by Experiment 1 was whether presenting pictures affected the shape of the value function for either type of outcome. In Figures 2A and 2B, the differences between the pics and neutral conditions appear to be small but consistent: The certain options for bears in the pics condition were seen as equivalent to risky options with larger negative outcomes for losses and larger positive outcomes for gains. This difference indicates that the average participant in the pics condition required a higher value from the risky option to switch for gains, and a lower one from the certain option to switch for losses (greater risk aversion and risk seeking, respectively). Like the $b$ parameter for the negative frame in the neutral condition, the $b$ parameter for framed losses of bears in the pics condition was significantly different from $1[t(26)=52.00, p<.001]$. The $b$ parameter for the pics condition was also significantly smaller than that for the neutral condition $[t(25)=6.56, p<.001]$. The func-
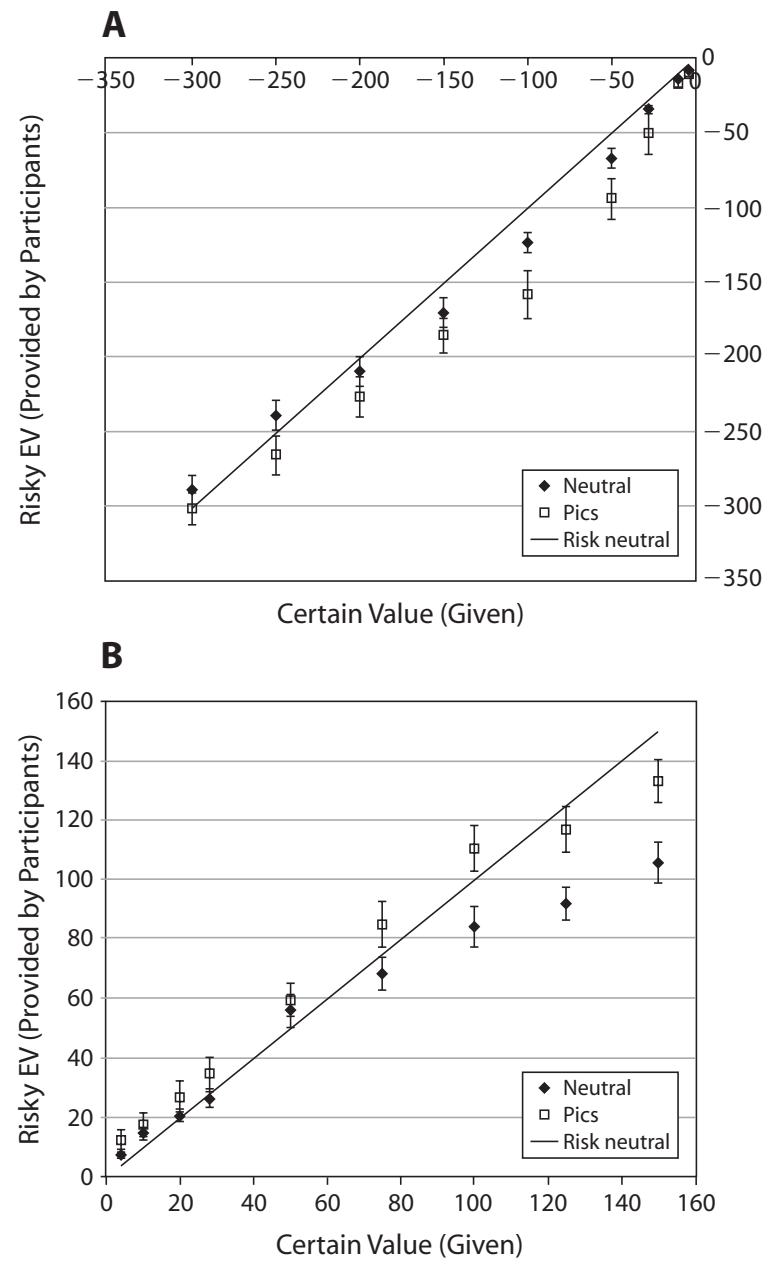

Figure 2. (A) Indifference points for the negative-frame/bears conditions. (B) Indifference points for the positive-frame/bears conditions. The solid line indicates risk neutrality. $\mathrm{EV}$, expected value. 


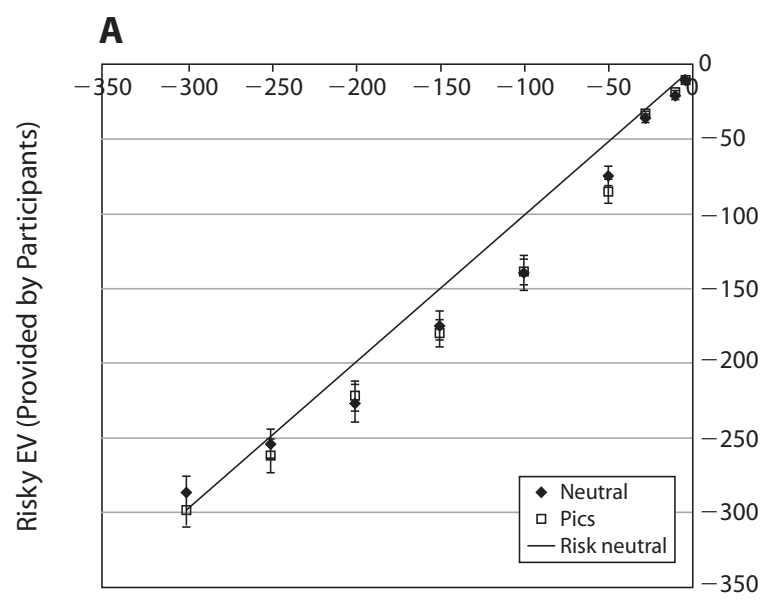

Certain Value (Given)

B

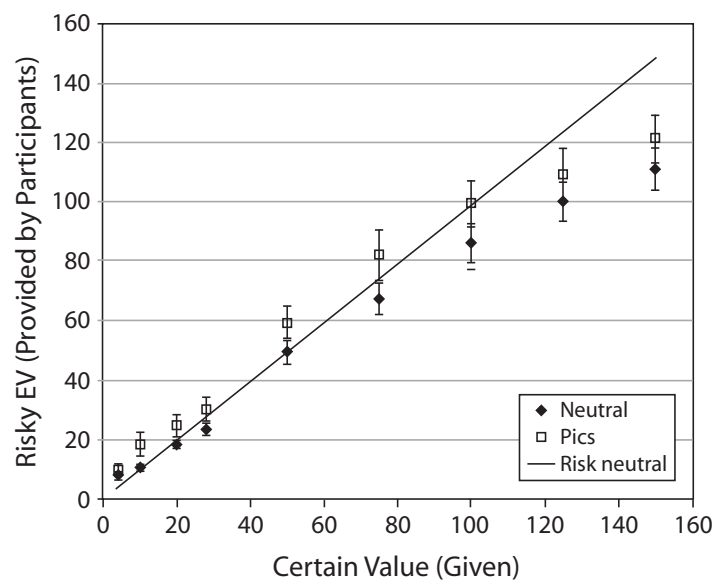

Figure 3. (A) Indifference points for the negative-frame/humanlife conditions. (B) Indifference points for the positive-frame/ human-life conditions. The solid line indicates risk neutrality. EV, expected value.

tion for framed losses for bears was significantly more nonlinear when participants saw pictures than when they saw colored squares. For framed gains for bears in the pics condition, the $b$ parameter was again significantly different from $1[t(25)=19.44, p<.001]$, but the difference in $b$ parameters for the pics and neutral conditions was not significant $[t(24)<1$, n.s.]. Presenting pictures to participants led to a more nonlinear function for framed losses of grizzly bears, but not for framed gains.

Examination of Figures $3 \mathrm{~A}$ and $3 \mathrm{~B}$ shows that pictures had a different effect on responses for human life than they did for bears. Responses for framed losses of human life showed almost no effect of pictures, with the data points for the negative-frame/pics condition and the negativeframe/neutral condition lying nearly on top of each other. However, for framed gains, the effect of pictures was similar to that for the grizzly bear choices, with participants who saw pictures demanding risky options with higher values to switch from the certain options. For human life in the pics condition, the $b$ parameter for the negative frame function was significantly different from $1[t(26)=33.95$, $p<.001]$. The $b$ parameters for the negative-frame/pics condition and the negative-frame/neutral condition were not significantly different for human life. Viewing pictures did not increase the nonlinearity of the framed loss function for human life. The $b$ parameter for framed gains in the pics condition was again significantly different from 1 $[t(25)=17.70, p<.001]$ and the difference between the $b$ parameters for the positive-frame/pics and positive-frame/ neutral conditions was significant $[t(24)=3.02, p<.01]$. The function for framed gains for human life was more nonlinear when pictures were shown.

\section{Discussion}

Both types of outcomes in Experiment 1 produced nonlinear value functions for framed gains and losses. The findings for framed losses were consistent with more valuable outcome types' having more nonlinear value 
Iable 2

Descriptive Statistics for Experiment 1

\begin{tabular}{|c|c|c|c|c|c|c|c|c|c|}
\hline \multirow[b]{4}{*}{ Frame } & \multirow{4}{*}{$\begin{array}{c}\text { Certain } \\
\text { Value }\end{array}$} & \multicolumn{8}{|c|}{ Average Risky EV } \\
\hline & & \multicolumn{4}{|c|}{ Neutral Condition } & \multicolumn{4}{|c|}{ Pics Condition } \\
\hline & & \multicolumn{2}{|c|}{ Bears } & \multicolumn{2}{|c|}{ Humans } & \multicolumn{2}{|c|}{ Bears } & \multicolumn{2}{|c|}{ Humans } \\
\hline & & $M$ & $S E$ & $M$ & $S E$ & $M$ & $S E$ & $M$ & $S E$ \\
\hline Negative & $\begin{array}{r}-4 \\
-10 \\
-28 \\
-50 \\
-100 \\
-150 \\
-200 \\
-250 \\
-300\end{array}$ & $\begin{array}{r}-8.79 \\
-14.79 \\
-33.79 \\
-67.18 \\
-123.33 \\
-170.32 \\
-209.68 \\
-238.91 \\
-288.65\end{array}$ & $\begin{array}{r}1.20 \\
0.99 \\
2.00 \\
6.45 \\
6.49 \\
10.05 \\
9.83 \\
9.54 \\
9.42\end{array}$ & $\begin{array}{r}-10.15 \\
-20.56 \\
-35.82 \\
-74.49 \\
-139.42 \\
-175.00 \\
-226.86 \\
-254.17 \\
-286.73\end{array}$ & $\begin{array}{r}2.01 \\
2.68 \\
2.61 \\
6.39 \\
11.65 \\
6.67 \\
12.50 \\
9.88 \\
11.28\end{array}$ & $\begin{array}{r}-10.48 \\
-16.98 \\
-50.64 \\
-94.07 \\
-158.27 \\
-185.56 \\
-226.79 \\
-265.86 \\
-301.60\end{array}$ & $\begin{array}{r}1.84 \\
2.30 \\
13.58 \\
13.40 \\
16.07 \\
11.81 \\
13.35 \\
12.87 \\
10.87\end{array}$ & $\begin{array}{r}-10.36 \\
-18.83 \\
-33.35 \\
-84.51 \\
-138.95 \\
-179.94 \\
-221.91 \\
-262.10 \\
-298.83\end{array}$ & $\begin{array}{r}1.49 \\
1.78 \\
2.10 \\
8.30 \\
8.75 \\
9.15 \\
10.20 \\
11.23 \\
10.55\end{array}$ \\
\hline Positive & $\begin{array}{r}4 \\
10 \\
20 \\
28 \\
50 \\
75 \\
100 \\
125 \\
150 \\
\end{array}$ & $\begin{array}{r}7.34 \\
14.62 \\
20.54 \\
26.09 \\
55.90 \\
68.23 \\
84.23 \\
91.83 \\
105.67 \\
\end{array}$ & $\begin{array}{l}0.89 \\
2.06 \\
1.85 \\
2.67 \\
5.59 \\
5.52 \\
6.76 \\
5.41 \\
6.90 \\
\end{array}$ & $\begin{array}{r}7.86 \\
10.77 \\
18.45 \\
23.50 \\
49.83 \\
67.50 \\
86.50 \\
100.43 \\
111.30 \\
\end{array}$ & $\begin{array}{l}1.36 \\
1.10 \\
1.43 \\
2.08 \\
4.31 \\
5.32 \\
6.74 \\
6.74 \\
7.08 \\
\end{array}$ & $\begin{array}{r}12.51 \\
17.63 \\
26.90 \\
34.79 \\
59.62 \\
84.81 \\
110.38 \\
116.86 \\
133.17 \\
\end{array}$ & $\begin{array}{l}3.32 \\
4.00 \\
5.34 \\
5.46 \\
5.50 \\
7.54 \\
7.72 \\
7.37 \\
7.22 \\
\end{array}$ & $\begin{array}{r}9.88 \\
18.33 \\
24.68 \\
30.27 \\
59.29 \\
82.31 \\
99.65 \\
109.62 \\
121.54 \\
\end{array}$ & $\begin{array}{l}2.00 \\
3.89 \\
3.65 \\
3.99 \\
5.73 \\
8.57 \\
7.81 \\
8.83 \\
8.08 \\
\end{array}$ \\
\hline
\end{tabular}

functions: The function for humans was more nonlinear than that for bears. However, the value function for framed gains was significantly less nonlinear for human life than for bears. The failure to find a consistent difference in nonlinearity of value functions for bears and human life suggests that nonlinearity may not vary across more and less valuable outcome types as proposed by Levin and Chapman (1990). The effect of pictures and names on nonlinearity was consistent: The negative frame function for bears and the positive frame function for human life were more nonlinear when participants saw pictures and names. This evidence refutes the idea that the results for human life seen in Bloomfield (2006) were due to an effect of pictures and names on the shape of the value function: Greater nonlinearity in the positive frame would lead to greater risk aversion in this frame, whereas a preference for the risky option was seen. Some other factor, such as $\mathrm{AL}$, is needed to explain these effects.

The finding that the value function for framed losses was more nonlinear for humans than for bears agrees with the findings of Bloomfield (2006). Participants in this study were risk averse in both frames for animal life when no pictures were shown; framing effects for animal life were found only in the pictures and names condition. The change between the two conditions was a change in

Table 3

Stevens's Law Parameters for Each Function in Experiment 1

\begin{tabular}{lcc}
\hline \multicolumn{1}{c}{ Function } & $a$ Parameter & $b$ Parameter \\
\hline Neutral, bears, framed losses & -2.897 & .806 \\
Neutral, humans, framed losses & -4.123 & .748 \\
Neutral, bears, framed gains & 2.865 & .724 \\
Neutral, humans, framed gains & 1.839 & .826 \\
Pics, bears, framed losses & -5.994 & .688 \\
Pics, humans, framed losses & -3.978 & .759 \\
Pics, bears, framed gains & 3.274 & .745 \\
Pics, humans, framed gains & 3.317 & .726 \\
\hline
\end{tabular}

the way participants made choices for framed losses. The findings discussed here suggest that the value function for animals is significantly less nonlinear than that for humans in the negative frame only and that presenting pictures and names leads to significantly greater nonlinearity in the negative frame only. The findings for animal life in Bloomfield (2006) may be explained by a combination of differences in the nonlinearity of the value function for animals and humans and by the way pictures and names affect nonlinearity.

\section{EXPERIMENT 2}

AL offers another explanation for why framing effects are not found with certain types of outcomes and can explain cases where a preference for the same option is shown in both frames. Experiment 2 examined the effect of an AL on preferences for framed losses and gains.

\section{Method}

Participants. Seventy-two undergraduates participated in this study for partial course credit. The data from two additional participants were excluded because they adopted a strategy like those mentioned in the Method section of Experiment 1.

Materials and Procedure. Experiment 2 used a methodology very similar to that of Experiment 1. Six additional levels of value for certain options were included, and participants responded to only grizzly bear or human life items. Of the 72 participants included in the analyses, 18 were in the negative-frame/bears condition, 21 addressed the negative-frame/human-life condition, 16 received the positive-frame/bears condition, and 17 received the positive-frame/ human-life condition. Participants in Experiment 2 were given an $\mathrm{AL}$ as part of their instructions. The presentation of the $\mathrm{AL}$ for framed gains was as follows:

For this group of 600 people [grizzly bears], a certain number is optimal for the group as a whole to exist. If less than 100 people/bears remain, the future outlook for the group as a whole is less favorable. 
A summary of this information was also presented at the top of each choice screen.

For framed losses, the manipulation wording was identical, except that it read "less than 400 people [bears] remain" and "at least 400 survive." The difference in the AL for framed losses and gains was necessary, because the outcomes were framed outcomes and not actual gains and losses. Although most of the certain outcomes had the same magnitude across the frames, because they were framed gains and losses the absolute number of group members still present in the group differed (e.g., a loss of 4 bears actually translated into a nonloss of 596 grizzly bears, whereas the same value for gains was only 4 bears saved). Because analyses for framed gains and losses were performed separately, the different ALs for the two frames were not problematic.

To increase the chances of seeing risk aversion for framed gains and risk seeking for framed losses for bears, all participants were presented with pictures and names.

\section{Results}

Figures 4A and 4B display results for gains and losses, respectively. AL is indicated by a dashed line and the solid line represents perfectly risk-neutral responses. Note that the dashed line for framed gains represents the point at which the AL is met, and the line for framed losses indicates the point at which the AL is violated. For both frames, the introduction of an AL affected preferences in the manner predicted: There was a rapid change in subjective value at the AL for framed gains, and, to a lesser degree, immediately after the AL for framed losses. For framed gains, the responses immediately before the AL were risk seeking and became risk averse after the $\mathrm{AL}$ was met. For losses, attitudes were risk seeking prior to the AL's being violated (i.e., when this level is still met) and became risk averse as the AL approached and after it had been crossed. Table 4 displays the average indifference points and standard errors for each frame and type of life.

Analyses included a total of 16 responses for each participant. ${ }^{7}$ Each response was coded as risk seeking or risk averse on the basis of its difference from the value of the certain option. The average number of risk-seeking

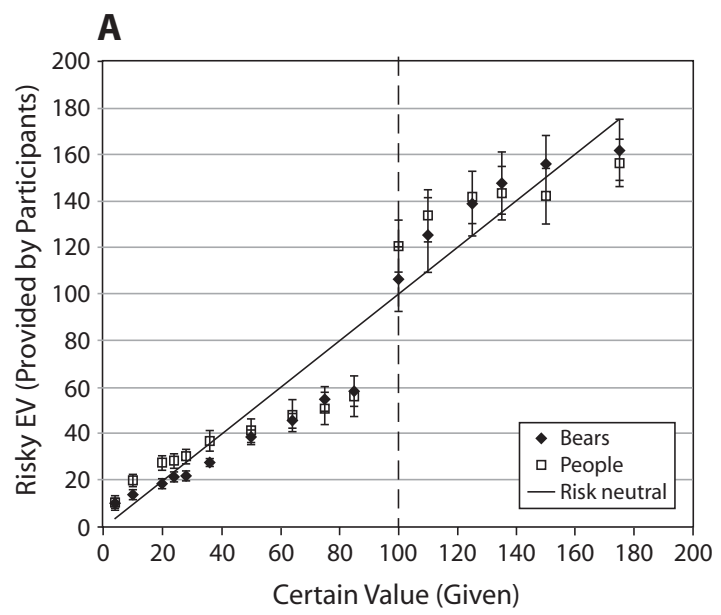

\section{B}

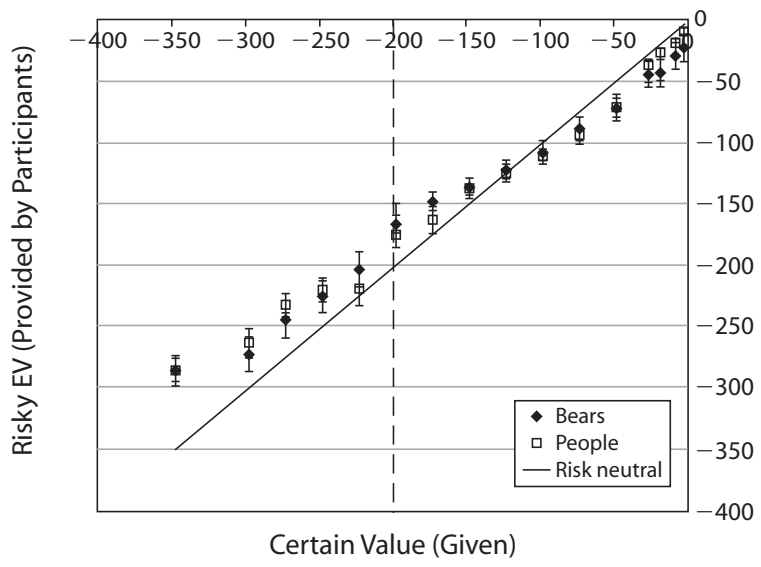

Figure 4. (A) Indifference points for framed gains of human lives and grizzly bears with an AL of "at least 100 survive." (B) Indifference points for framed losses of human lives and grizzly bears with an AL of "no more than 200 lost." The solid line indicates risk neutrality, and the dashed line indicates the AL. EV, expected value. 
responses was tallied separately for each participant for certain options that met the AL and for those that did not meet the AL. Table 5 displays the average proportion of risky choices for each life $\times$ frame condition.

For the negative-frame/bears condition, the difference in the proportion of risk-seeking responses was significant $[t(17)=4.49, p<.001]$ : Participants were more risk seeking on average when the certain option met the AL. A similar pattern was found for the negative-frame/humanlife condition $[t(20)=4.67, p<.001]$. The AL led to a similar change in risk attitudes for both the positiveframe/bears condition and the positive-frame/human-life condition: When the AL was met by the certain option, more choices were risk averse than risk seeking, and when the AL was not met, more choices were risk seeking than risk averse. The comparison for the positive-frame/bears condition was significant $[t(15)=3.57, p<.01]$, but the test for the positive-frame/human-life condition did not reach significance. This is due likely to the tendency for risk-averse responses for the smaller certain values $(4,10$, 20,24 , and 28). When only the six values immediately below the AL were compared with the six values including and immediately above the AL, the difference in risk preferences for this condition was significant $[t(16)=2.30$, $p<.05]$, with greater risk seeking when the AL was not met. The reason behind the risk aversion for the smaller certain values is discussed below.

\section{Discussion}

The findings of Experiment 2 show that a salient AL can affect risk preferences for negatively and positively framed outcomes in ways that reverse the typical risk attitudes shown in each frame. In the area approaching the AL for framed gains, participants showed a risk-seeking tendency in their responses: Risky options that provided some chance of achieving the AL were preferred to certain options that would not meet it, even if these certain options had a higher expected value. After the AL was met by the certain option, participants showed risk aversion in their choices. In the area approaching the AL for framed losses (in this case, where the AL would no longer be met), participants became risk averse: Certain options that guaranteed that the AL would be met were preferred to risky options that might lead to the AL not being met, even if the certain option's expected value was worse. Smaller certain values for framed losses led to risk-seeking choices, but only when the nonzero outcome value of the risky option was lower than the AL: When the risky option's nonzero value would have violated the AL (resulting in a loss of more than 200 members of the group), participants preferred the certain option that met the AL. This indicates that participants were risk seeking for losses, but only to the point where the AL would still be met regardless of the outcome.

For human life in particular, participants showed risk aversion for the lower certain option values for framed gains. This pattern is inconsistent with a risk-seeking preference seen for framed gains when the AL was attainable only through choice of the risky option. However, the explanation for this anomaly is that participants were "hold-
Table 4

Descriptive Statistics for Experiment 2

\begin{tabular}{|c|c|c|c|c|c|}
\hline \multirow[b]{3}{*}{ Frame } & \multirow{3}{*}{$\begin{array}{c}\text { Certain } \\
\text { Value }\end{array}$} & \multicolumn{4}{|c|}{ Average Risky EV } \\
\hline & & \multicolumn{2}{|c|}{ Bears } & \multicolumn{2}{|c|}{ Humans } \\
\hline & & $M$ & $S E$ & $M$ & $S E$ \\
\hline Negative & $\begin{array}{r}-4 \\
-10 \\
-20 \\
-28 \\
-50 \\
-75 \\
-100 \\
-125 \\
-150 \\
-175 \\
-200 \\
-225 \\
-250 \\
-275 \\
-300 \\
-350\end{array}$ & $\begin{array}{r}-22.81 \\
-29.74 \\
-43.33 \\
-44.89 \\
-71.85 \\
-88.89 \\
-108.33 \\
-122.22 \\
-136.48 \\
-148.33 \\
-166.67 \\
-203.61 \\
-225.46 \\
-244.44 \\
-272.50 \\
-285.65\end{array}$ & $\begin{array}{r}11.10 \\
10.80 \\
11.42 \\
10.11 \\
10.57 \\
9.27 \\
9.36 \\
7.59 \\
6.92 \\
7.46 \\
7.18 \\
14.44 \\
12.94 \\
15.05 \\
13.96 \\
12.17\end{array}$ & $\begin{array}{r}-9.98 \\
-19.60 \\
-27.10 \\
-37.03 \\
-71.35 \\
-94.52 \\
-111.83 \\
-124.84 \\
-137.54 \\
-163.25 \\
-175.63 \\
-218.49 \\
-220.24 \\
-232.94 \\
-263.41 \\
-285.40\end{array}$ & $\begin{array}{r}2.33 \\
3.42 \\
3.51 \\
4.14 \\
7.99 \\
6.83 \\
6.20 \\
6.97 \\
8.65 \\
11.04 \\
10.07 \\
14.14 \\
9.16 \\
9.45 \\
11.67 \\
9.64\end{array}$ \\
\hline Positive & $\begin{array}{r}4 \\
10 \\
20 \\
24 \\
28 \\
36 \\
50 \\
64 \\
75 \\
85 \\
100 \\
110 \\
125 \\
135 \\
150 \\
175\end{array}$ & $\begin{array}{r}10.08 \\
13.63 \\
18.35 \\
21.17 \\
21.75 \\
27.52 \\
38.44 \\
45.50 \\
54.69 \\
58.13 \\
106.30 \\
125.21 \\
138.65 \\
147.50 \\
155.78 \\
161.77\end{array}$ & $\begin{array}{r}2.15 \\
2.29 \\
2.14 \\
2.02 \\
1.96 \\
1.60 \\
3.23 \\
3.15 \\
5.29 \\
6.48 \\
13.87 \\
15.99 \\
13.71 \\
13.23 \\
12.05 \\
13.13\end{array}$ & $\begin{array}{r}10.17 \\
19.64 \\
27.21 \\
28.19 \\
30.05 \\
36.64 \\
41.03 \\
47.66 \\
50.64 \\
55.93 \\
120.44 \\
133.53 \\
141.32 \\
143.19 \\
141.96 \\
156.13\end{array}$ & $\begin{array}{r}2.83 \\
2.54 \\
3.13 \\
3.17 \\
2.98 \\
4.36 \\
5.27 \\
6.93 \\
7.07 \\
8.74 \\
11.19 \\
11.16 \\
11.18 \\
11.43 \\
11.90 \\
10.00\end{array}$ \\
\hline
\end{tabular}

ing out" for a risky option whose nonzero outcome would meet the AL: The expected value for the risky option for small values of framed gains exceeded the value of the certain option, making these choices risk averse. As the AL approached, participants still showed preference for the risky option with a nonzero outcome that would meet the AL, but this option then had lower expected value than the value of the certain option, making the participants' choices risk seeking.

Another unexpected finding is that participants remained risk averse for framed losses after the AL was violated: Why did participants not become extremely risk seeking, preferring the risky option over the certain option at every choice, because only the risky option offered a chance that the AL could be met? One possibility

Table 5

Average Proportion of Risk-Seeking Choices Made in Experiment 2 for Each Type of Life in Each Frame

\begin{tabular}{cccccc}
\hline & \multicolumn{4}{c}{ Certain Option } \\
\cline { 2 - 3 } \cline { 5 - 6 } Frame & \multicolumn{2}{c}{ Satisfies AL } & & Does Not Meet AL \\
\cline { 2 - 3 } \cline { 5 - 6 } & Bears & People & & Bears & People \\
\hline Positive & .43 & .42 & & .73 & $.52(.69)^{*}$ \\
Negative & .54 & .52 & & .22 & .20 \\
\hline
\end{tabular}

*Average proportion of risky choices including only the six certain options immediately below the AL. 
is that participants responding to losses came to ignore the common outcome (1/3 chance of none dying) in their choices and instead focused on the nonzero outcome. This idea is supported by the finding that, at the group level, the risky option was never preferred to the certain option with equal expected value if the risky option's nonzero outcome would not meet the AL. Another likely reason for this behavior was the nature of the task: Participants who selected the risky option were then presented with another risky option with a worse outcome. If they selected the risky option again, the outcome again was worse in the next choice. Even with the lack of a sure avenue to reach their goal, participants may have felt that selecting the certain option was preferable to making further choices toward ever-worsening risky options, increasing the potential damage that might be done.

\section{GENERAL DISCUSSION}

The findings of Experiments 1 and 2 indicate that both the $\mathrm{AL}$ and shape of the value function can contribute to outcome type effects such as those found by Bloomfield (2006), Levin and Chapman (1990), Schneider (1992), and Wang and Johnston (1995). The findings of Experiment 1 show that some differences in nonlinearity do exist between outcome types and that a manipulation that encourages framing effects (presenting pictures and names of the victims; Bloomfield, 2006) increases the nonlinearity of the value function, which should increase the propensity for framing effects. Experiment 2 demonstrated that an $\mathrm{AL}$ could change risk preferences for both framed gain and loss outcomes, such that participants showed risk seeking for framed gains and risk aversion for framed losses. Together, these factors can explain an absence of framing effects without a predominant preference in either frame (through a less nonlinear value function) and an absence of framing effects when there is a strong preference for the same option in both frames (through an AL that makes one of the options particularly attractive or unattractive).

Experiment 1 revealed significantly nonlinear value functions for both human and animal life with no picture manipulation. This finding challenges the argument that people do not show framing effects for less valuable outcome types (e.g., animals) because they have nearly linear value functions for these outcome types. Although some differences in nonlinearity of the value function for animal and human life were found, they were not consistent: For framed losses, the value function for human life was more nonlinear than that for bears, but the function for framed gains of human life was less nonlinear than that for bears. It seems that for this particular contrast of outcome types (humans vs. bears), the less valuable outcome type neither has a strictly near linear value function nor consistently lags behind the more valuable outcome type in degree of nonlinearity. Pictures and names had a consistent effect on the shape of the value function, however, with greater nonlinearity seen for the pics condition whenever there was a significant difference. Presenting pictures and names of the victims should encourage framing effects by strengthening the risk attitude engendered by the positive and negative frames.

Experiment 2 showed that giving an AL to participants can lead them to show both risk seeking and risk aversion, regardless of frame. For framed gains, when the certain option met the AL, participants preferred it to a risky option of equal expected value. For framed losses, participants preferred the certain option over the risky option if the certain option met the AL and the risky option's nonzero outcome would violate the AL. These results demonstrate that a preference for the certain option can arise in both frames if participants have a relatively low AL (e.g., at or below 200). Similarly, Experiment 2 found that when the certain option did not meet the AL, participants preferred the risky option (as long as its nonzero outcome allowed a chance of attaining the AL) in the positive frame. This result shows that an AL higher than the certain option's value can result in risk seeking in the positive frame for scenarios like the Asian Disease problem (which offers a risky option with a nonzero outcome of everyone's being saved). Risk seeking was not found in the negative frame when certain options did not meet the AL, however: Participants preferred the risky option only to the extent that its nonzero outcome value would not violate their $\mathrm{AL}$ and were risk averse otherwise. The methodology used in Experiment 2, where the risky option for framed losses became worse each time it was chosen, likely led to the risk aversion seen here. In one-shot choice situations like the Asian Disease problem, participants with an AL that the certain option did not meet would likely prefer the risky option. If this is the case, the presence of a relatively high AL could explain cases where risk seeking is shown in both frames, such as for human life when pictures and names of the victims are shown (Bloomfield, 2006) or when group size is small (Wang \& Johnston, 1995).

Schneider (1992) argued that AL alone can account for framing effects. However, the findings of Experiment 2 indicate that risk aversion for gains and risk seeking for losses are not AL-dependent phenomena: Participants were risk seeking for losses when the AL could be met by the certain option, and they were risk averse for gains when the AL could possibly be met by the risky option. Although the latter case accords with Schneider's argument that a desire for security (low risk) guides most decision makers, the former demonstrates that participants are risk seeking for losses even when it is not necessary to meet their AL. The value function's shape contributes to the preference for the risky option in the negative frame. Furthermore, for AL alone to account for framing effects, participants must adopt an AL for all scenarios that produce framing effects, and the level of this AL must be met by the certain option in the positive frame and not met in the negative frame. Preliminary work on individual ALs for animal and human life indicates that the size of the AL adopted in the positive frame does not differ from that adopted in the negative frame (Bloomfield, 2005). There is also no reason to assume that an $\mathrm{AL}$ is formed for every framed scenario, so it is unlikely that differing ALs in the loss and gain frames are the main cause of the framing effect. 
The results of Experiments 1 and 2 illuminate the results seen in Bloomfield (2006) and indicate that a combination of differences in the value function and ALs is implied by these results. Preferences for animal lives in Bloomfield (2006) when no pictures were shown are consistent with results from Experiment 2 when participants chose between a certain option that met their AL and a risky option that might not meet it. If participants did have an AL lower than 200 for animal lives in Bloomfield (2006), it would have led to the preference for the certain option in both frames that was seen. The shape of the value function for framed losses should also have been less nonlinear in this case (at least, less nonlinear than that for humans), decreasing the extent to which this frame encouraged a risk-seeking attitude. The introduction of pictures and names, according to the results of Experiment 1 , would have increased the nonlinearity of the value function for framed losses. However, to lead to the framing effect found in Bloomfield (2006), pictures and names would also have had to raise the AL to a level less easily satisfied by the certain option, allowing the risk attitude to have a greater impact. The idea that the pictures manipulation might increase the $\mathrm{AL}$ is consistent with the results for human lives in Bloomfield (2006): In order to explain the risk seeking shown in both frames when this manipulation was presented, the AL would have had to be increased beyond the value of the certain option.

Although Experiment 2 found that inducing an $\mathrm{AL}$ affected risk preferences, it did not investigate whether participants form an AL for a particular outcome type on their own. The domain of life-and-death decisions, however, does seem to be ripe for creating ALs. As Wang (1996) suggested, participants may be motivated to save all humans at risk under certain circumstances (e.g., for very small groups). When group members are viewed as less unique and individually valuable, such as in a group of animals, the desire to maintain a breeding population may lead an AL for saving/not losing animal lives that is lower; to the extent that the AL is lower than the value of the certain option, this would increase preference for the certain option. For example, in Schneider (1992) the animal scenario that yielded preference for the certain option in both frames involved the last 1,200 members of a species, circumstances in which maintaining a breeding population would be of crucial importance. How an $\mathrm{AL}$ is formed should be investigated more directly using different outcome types and different group sizes. The effect of a high $\mathrm{AL}$ on responses to framed losses in the standard Asian Disease problem format (i.e., a one-shot choice between one risky and one certain option) also needs to be investigated. Although Experiment 2 failed to find risk seeking for framed losses when the AL was not met by the certain option, this is likely due to the methodology used to measure indifference points.

The studies discussed here extend the work on outcome type and frame interactions in several ways. First, a direct examination of how the value function differs between outcome types was made possible by mapping out the functions. Previous work highlighting the role of the value function in framing effects, such as Levin and
Chapman (1990), has not explored whether this factor actually differs among outcome types. Furthermore, the evidence provided for how AL influences the shape of the value function broadens the explanatory power of current theories by allowing not only for the prediction of when framing effects will occur, but also the direction of preferences when the effect is not shown. Situations where frame fails to sway choice may not be due to a failure to affect risk attitude, but rather due to the risk attitude becoming less important in the face of a more pressing reason for choice.

\section{AUTHOR NOTE}

This research was part of the doctoral dissertation of the author. The author thanks Douglas Medin, Lance Rips, and Reid Hastie for their comments on an earlier version of this work and Sergey Blok for his comments on the final version. Correspondence concerning this article should be addressed to A. N. Bloomfield, Department of Psychology, DePaul University, 409 Byrne Hall, 2219 N. Kenmore Ave., Chicago, IL, 60614 (e-mail: abloomfi@depaul.edu).

\section{REFERENCES}

Bloomfield, A. (2005). [Aspiration levels for framed scenarios]. Unpublished raw data.

Bloomfield, A. (2006). Group size and the framing effect: Threats to human beings and animals. Memory \& Cognition, 34, 929-937.

Fagley, N. S., \& Miller, P. M. (1997). Framing effects and arenas of choice: Your money or your life? Organizational Behavior \& Human Decision Processes, 71, 355-373.

Kahneman, D., \& TVersky, A. (1979). Prospect theory: An analysis of decision under risk. Econometrica, 47, 263-292.

Kahneman, D., \& Tversky, A. (1984). Choice, values, and frames. American Psychologist, 39, 341-350.

LeVin, I. P., \& Chapman, D. P. (1990). Risk taking, frame of reference, and characterization of victim groups in AIDS treatment decisions. Journal of Experimental Social Psychology, 26, 421-434.

Lopes, L. L. (1987). Between hope and fear: The psychology of risk. In L. Berkowitz (Ed.), Advances in experimental social psychology (Vol. 20, pp. 255-295). San Diego: Academic Press.

SCHNEIDER, S. L. (1992). Framing and conflict:Aspiration level contingency, the status quo, and current theories of risky choice. Journal of Experimental Psychology: Learning, Memory, \& Cognition, 18, 1040-1057.

SmidTs, A. (1997). The relationship between risk attitude and strength of preference: A test of intrinsic risk attitude. Management Science, 43, 357-370.

Stevens, S. S. (1961). To honor Fechner and repeal his law: A power function, not a log function, describes the operating characteristic of a sensory system. Science, 133, 80-86.

Torgerson, W. S. (1958). Theory and methods of scaling. New York: Wiley.

TVERsky, A., \& Kahneman, D. (1981). The framing of decisions and the psychology of choice. Science, 211, 453-458.

WANG, X. T. (1996). Domain-specific rationality in human choices: Violations of utility axioms and social contexts. Cognition, 60, 31-63.

WAng, X. T., \& Johnston, V. S. (1995). Perceived social context and risk preference: A re-examination of framing effects in a life-death decision problem. Journal of Behavioral Decision Making, 8, 279-293.

WANG, X. T., Simons, F., \& Bredart, S. (2001). Social cues and verbal framing in risky choice. Journal of Behavioral Decision Making, 14, $1-15$.

\section{NOTES}

1. Nonlinearity in the value function is measured separately for gains and losses. The value function could be perfectly linear within outcome domain, but still be nonlinear in its entirety if the slope for losses differed from that for gains (thanks to an anonymous reviewer for bringing up this point). Such a difference is likely given loss aversion (Kahneman $\&$ Tversky, 1984). However, the question of nonlinearity in the value 
function as it is applied to framing effects pertains to the function within a particular outcome domain.

2. Schneider (1992) explained that security and potential motivations cannot play a role in framing effects because the outcomes in the two frames are objectively identical, and so a person with a given level of security mindedness and potential mindedness would have the same preference ordering in both frames.

3. Schneider (1992) found that across several scenarios participants tended to have more inconsistent preferences in the negative frame, rather than show a strong preference for the risky option. As such, she argued that participants are torn between the preference for security, which favors the certain option, and their AL, which rejects the certain option.

4. Kahneman and Tversky (1979) mentioned circumstances in which the value function for gains might show a steep rise and the value function for losses a steep drop, when a particular value has qualitatively more impact (e.g., a loss that would cause the decision maker to move to another neighborhood). These circumstances could cause the value function for gains to become convex (indicating risk seeking) and the value function for losses concave (indicating risk aversion). However, the manner in which $\mathrm{AL}$ influences risk preferences has not been demonstrated.

5. Technically, because the functions assessed in Experiments 1 and 2 were assessed in the context of risk, they are utility functions, not value functions. Value functions must be mapped by examining judgments in situations that are risk free (such as asking a participant to name a midpoint between 600 lives and 200 lives; Torgerson, 1958). However, utility functions may be better predictors of risky decision making than value functions (Smidts, 1997), and Kahneman and Tversky (1979) argued that a function derived from risky choices (i.e., a utility function) should share the same characteristics as prospect theory's value function. For ease of applying their findings to past research, the term value function is used to describe the functions plotted for participants in Experiments 1 and 2.

6 . The data for the two highest levels of value ( 175 and 200 for framed gains, and 350 and 400 for framed losses) were excluded from analyses because more than half of participants chose the certain option in the gain frame and the risky option in the loss frame, after the value of the risky option reached 600 (its maximum value). Their indifference point for the value of the certain option may have been considerably higher than the expected value of the highest risky option, and the inclusion of these responses would have artificially flattened the top of the value function.

7. Unlike in Experiment 1, most participants' responses failed to reach the ceiling of 600 lives in the risky option for the value level of 175 for gains and 350 for losses, although many participants did still reach this level for a value of 200 for gains and 400 for losses.

(Manuscript received August 20, 2007; revision accepted for publication December 19, 2007.) 